\title{
Principais Técnicas Fisioterápicas em Cães: Revisão de Literatura
}

\author{
Main Physiotherapy Techniques in Dogs: Literature Review
}

Larissa Carolina Peregrino*a; Elaine Santana Gonçalves ${ }^{\mathrm{b}}$; Elizângela Guedes ${ }^{\mathrm{a}}$; Sávio Tadeu Almeida Júnior ${ }^{\mathrm{a}}$

\author{
${ }^{a}$ Centro Universitário do Sul de Minas. MG, Brasil. \\ bUniversidade José do Rosário Vellano, Programa de Pós-Graduação Stricto Sensu em Reprodução, Sanidade e Bem-Estar Animal. MG, Brasil. \\ *E-mail: larissa@unis.edu.br
}

\begin{abstract}
Resumo
É crescente na medicina veterinária as técnicas e especialidades voltadas ao aprimoramento e prestação de serviços que objetivam o bem-estar animal e qualidade de vida. Nesse sentido, a reabilitação animal busca a recuperação e reestabelecimento das funções do animal, ou de forma que fique mais próxima das condições normais, seja promovendo analgesia, resolução da inflamação, a melhoria na locomoção, bem como a redução do desconforto. A fisioterapia, por sua vez, é uma especialidade que desenvolve várias técnicas, podendo ser utilizada para promover tanto a estimulação local como generalizada. Pode também ser empregada como método conservador ou, ainda, ser associada aos métodos cirúrgicos. Apresenta-se, de maneira geral e sintetizada, conceitos, técnicas e a importância da reabilitação física animal, por meio de uma revisão bibliográfica, buscando realizar uma abordagem voltada às alterações ortopédicas, neurológicas e doenças degenerativas e/ou crônicas. Para isso, foi realizada uma revisão de literatura em bases de dados e livros publicados entre 2000 a 2020, tanto em Língua Inglesa como Portuguesa, com os seguintes descritores: cães, medicina veterinária, reabilitação, fisioterapia, fisiatria, controle da dor, ortopedia, medicina veterinária integrativa. A fisioterapia se demonstra benéfica no tratamento e prevenção de patologias ortopédicas, neurológicas e também no controle da obesidade canina. Várias técnicas e equipamentos podem ser empregados, sendo o protocolo terapêutico individual, devendo sempre ser elaborado com base nas necessidades e limitações de cada paciente. Para isso, é imprescindível que o médico veterinário fisiatra tenha pleno conhecimento das técnicas de reabilitação.
\end{abstract}

Palavras-chave: Reabilitação. Ortopedia. Atrofia. Medicina Integrativa.

\begin{abstract}
It is growing in veterinary medicine the techniques and specialties focused on the improvement and provision of services that aim to promote the animal's welfare and quality of life. Thus, animal rehabilitation seeks the recovery and reestablishment of the animal's functions, or in a way that is closer to normal conditions, by either promoting pain relief, reducing inflammation, improving locomotion, as well as reducing discomfort. Physiotherapy, in turn, is a specialty that develops various techniques, and can be used to promote both local and generalized stimulation. It can also be used as a conservative method or, still, be associated with surgical treatment. It is herein presented, in a general and synthesized way, concepts, techniques, and the importance of animal physical rehabilitation, by means of a bibliographic review, trying to make an approach focused on orthopedic and neurological alterations, and degenerative and/or chronic diseases. Therefore, a literature review was conducted in databases and books, published between 2000 and 2020, both in English and Portuguese, with the following descriptors: dogs, veterinary medicine, rehabilitation, physiotherapy, physiatry, pain control, orthopedics, integrative veterinary medicine. Physiotherapy has proven beneficial in the treatment and prevention of orthopedic and neurological pathologies, and also in the canine obesity control. Several techniques and equipment can be used, and the therapeutic protocol is individual, and should always be prepared based on the needs and limitations of each patient. For this, it is essential that the veterinary physiatrist has full knowledge of rehabilitation techniques.
\end{abstract}

Keywords: Rehabilitation. Orthopedics. Atrophy. Integrative Medicine.

\section{Introdução}

Crescem, na medicina veterinária, as demandas e as especialidades voltadas ao aprimoramento e prestação de serviços voltados ao bem-estar animal e longevidade (HUMMEL; VICENTE, 2019).

A reabilitação física é um processo que objetiva a recuperação, devolvendo a máxima forma ou função locomotora fisiológica, de modo a prevenir a incapacidade e atrofias após o animal ter uma lesão ou enfermidade. Como parte integrada desse processo, a fisioterapia se tornou a especialidade encarregada do tratamento de determinadas doenças, podendo ser aplicada como método conservador ou associada ao tratamento cirúrgico (DINIZ-GAMA, 2007).

A fisioterapia possui como finalidade diminuir as disfunções e promover analgesia, mantendo e promovendo a melhora da função e aptidão física, gerando bem-estar e qualidade de vida (GORDON-EVANS; KNAP; SCHULZ, 2014; ALVES; STURION; GOBETTI, 2019).

$\mathrm{O}$ presente estudo demonstra, de maneira geral e sintetizada, os conceitos, as modalidades e a importância da fisioterapia veterinária para a promoção da recuperação e da prevenção de patologias em animais de companhia. 


\section{Desenvolvimento}

Foi realizada uma revisão de literatura nas seguintes bases de dados: Elsevier, Google Academic, Periódicos Capes, Science Direct e Medline. Também foram selecionados livros. Para a seleção foram utilizados como critérios de inclusão estudos publicados entre 2000 a 2020, tanto em Língua Inglesa como Portuguesa, com os seguintes descritores: cães, medicina veterinária, reabilitação, fisioterapia, fisiatria, controle da dor, ortopedia, medicina veterinária integrativa. Foram excluídos trabalhos sem embasamento científico ou aqueles cujos dados não tenham sido disponibilizados por completo.

\subsection{A fisioterapia como técnica de reabilitação animal}

A fisioterapia visa o desenvolvimento de técnicas de estimulação local ou generalizada e seus benefícios já são amplamente difundidos em humanos. Atualmente, a medicina veterinária vem buscando conhecer mais os recursos dessa área com estudos e desenvolvimento de métodos para sua utilização em animais (ALVES; STURION; GOBETTI, 2019).

O uso da fisioterapia na reabilitação animal possui uma gama de finalidades. No Quadro1 são apresentadas as principais indicações e modalidades terapêuticas relatadas entre a literatura consultada.

Quadro 1 - Principais indicações e modalidades terapêuticas relatadas entre a literatura consultada

\begin{tabular}{|c|c|}
\hline Indicações & Modalidades terapêuticas \\
\hline $\begin{array}{c}\text { Controle da inflamação e } \\
\text { edema }\end{array}$ & $\begin{array}{c}\text { Crioterapia, bandagem } \\
\text { compressiva, massagem. }\end{array}$ \\
\hline $\begin{array}{l}\text { Aumento ou preservação da } \\
\text { amplitude de movimento }\end{array}$ & $\begin{array}{l}\text { Alongamento, ultrassom } \\
\text { terapêutico, massagem }\end{array}$ \\
\hline Lesões em tecidos moles & $\begin{array}{c}\text { Crioterapia, ultrassom } \\
\text { terapêutico, laserterapia, } \\
\text { cinesioterapia, alongamento, } \\
\text { hidroterapia }\end{array}$ \\
\hline Controle da dor & $\begin{array}{c}\text { Crioterapia, laserterapia, } \\
\text { TENS*, massagem, } \\
\text { magnetoterapia. }\end{array}$ \\
\hline Afecções articulares & $\begin{array}{c}\text { Crioterapia, massagem, } \\
\text { alongamento, cinesioterapia, } \\
\text { hidroterapia, ente outros } \\
\text { a depender da articulação } \\
\text { envolvida. }\end{array}$ \\
\hline Reparação de fraturas & $\begin{array}{c}\text { Crioterapia, ultrassom } \\
\text { terapêutico, laserterapia, } \\
\text { magnetoterapia, FES*, } \\
\text { Cinesioterapia, exercícios } \\
\text { proprioceptivos. }\end{array}$ \\
\hline Afecções em coluna vertebral & $\begin{array}{l}\text { Crioterapia, massagem, } \\
\text { alongamento passivo, } \\
\text { cinesioterapia, estimulação } \\
\text { elétrica neuromuscular, } \\
\text { exercícios terapêuticos e } \\
\text { proprioceptivos, hidroterapia, } \\
\text { caminhada. }\end{array}$ \\
\hline Fortalecimento muscular & $\begin{array}{l}\text { NMES*, hidroterapia, } \\
\text { cinesioterapia }\end{array}$ \\
\hline
\end{tabular}

\begin{tabular}{|c|c|}
\hline Indicações & Modalidades terapêuticas \\
\hline Lesões neurológicas & $\begin{array}{c}\text { Cinesioterapia, laserterapia, } \\
\text { eletroestimulação, exercícios } \\
\text { proprioceptivos, laserterapia }\end{array}$ \\
\hline Cicatrização tecidual & Laserterapia \\
\hline Sistema Respiratório & $\begin{array}{c}\text { Compressão-descompressão } \\
\text { torácica súbita, estimulação } \\
\text { costal. }\end{array}$ \\
\hline Cinomose & $\begin{array}{c}\text { Laserterapia, cinesioterapia, } \\
\text { magnetoterapia, eletroterapia } \\
\text { e hidroterapia }\end{array}$ \\
\hline Controle e redução obesidade & $\begin{array}{c}\text { Exercícios, hidroterapia, } \\
\text { manejo alimentar. }\end{array}$ \\
*TENS: Transcutaneal electrical & nerve stimulation; NMES:
\end{tabular}

Neuro muscular electrical stimulation; FES: Functional electrical stimulation.

Fonte: dados da pesquisa.

Em pacientes com déficits neurológicos se objetiva com a fisioterapia a recuperação dos tecidos nervosos lesionados, prevenir ou reduzir o desenvolvimento da atrofia muscular, melhorar a função dos membros parésicos e/ou paralisados e prevenir ou tratar o desenvolvimento de contraturas e de fibrose nos tecidos moles (GORDON-EVANS; KNAP; SCHULZ, 2014).

Da mesma forma como ocorre com os humanos, os animais idosos podem apresentar alterações como redução da massa muscular, comprometimento da função articular e redução da propriocepção, em decorrência de distúrbios ortopédicos e neurológicos, ou até mesmo em animais clinicamente saudáveis. Nesse sentido, a fisioterapia oferece uma grande variedade de modalidades que podem ser usadas para neutralizar esses problemas, tais como: esteira subaquática, exercícios ativos, pistas de propriocepção, utilização de exercícios de equilíbrio e obstáculos (MUCHA; BOCKSTAHLER, 2019).

Dentro de um programa de redução de peso, os exercícios fisioterápicos têm sido amplamente recomendados e empregados, em especial, os exercícios de baixo impacto utilizando esteiras subaquáticas associadas a um programa controlado de exercícios domésticos, como natação e caminhadas com guia (MUCHA; BOCKSTAHLER, 2019).

Assim, diversas técnicas de fisioterapia e reabilitação podem ser empregadas. Entretanto, se deve ter em mente que um protocolo terapêutico é individual, devendo serem respeitadas as necessidades do paciente, suas indicações e possibilidades de efetivo sucesso, assim como as contraindicações e desvantagens do tratamento. Um bom plano terapêutico deve buscar o resultado mais rápido e positivo ao cão para atender o objetivo de máxima recuperação da função, prevenir a incapacidade permanente, sequelas e lesões futuras (ALVES; STURION; GOBETTI, 2019).

\subsection{Avaliação do paciente}

A semiologia é fundamental para o início da elaboração de um plano terapêutico. Uma boa anamnese em conjunto com o histórico do animal se faz necessário, a fim de se conhecer 
a condição antes e após a afecção (HUMMEL; VICENTE, 2019).

No Quadro 2 são apresentadas algumas perguntas pertinentes para a condução da anamnese.

Quadro 2 - Guia com sugestões para a condução de anamnese ortopédica e fisioterápica

Início e evolução da doença:

Quando o problema teve início?

É recente ou crônico?

Está ocorrendo progressão ou estabilização do quadro?

Há situações ou períodos de melhora ou piora?

Ambiente:

Tipo de piso em que o animal passa a maior parte do tempo?

Presença de escadas, rampas ou obstáculos?

Acesso à rua?

Sobe em superfícies elevadas (cama ou sofá)?

Manejo:

Quais tipos de brincadeiras?

Hábitos de vida (animal ativo, restrição de espaço físico, passeios)?

Qual a função do animal (trabalho, guarda ou companhia)? Frequência de exercícios?

Histórico sanitário:

Ocorrência de traumatismos, quedas ou atropelamento?

Doenças concomitantes ou degenerativas?

Tratamentos anteriores ou procedimentos cirúrgicos?

Dieta:

Tipo, qualidade e frequência de alimentação?

Recebe petiscos?

Mudança de comportamento e de postura:

Houve mudanças de comportamento, de personalidade ou convulsões?

Animal mais agressivo ou quieto?

Dificuldade para levantar, deitar, saltar ou executar

algum movimento? Animal tropeça? Se sim, qual membro

acometido?

Sente dor? Se sim, qual local e quando?

Fonte: Dados da pesquisa.

$\mathrm{O}$ exame físico deve ser realizado abrangendo tanto a inspeção dinâmica como a estática. $\mathrm{Na}$ inspeção dinâmica, com o animal em movimento, deve ser observada a deambulação e a presença de claudicação e/ou compensações. Já com o animal em repouso (estática) se procura verificar a presença de assimetrias, diferenças de volume, deformidades, descolorações e/ou presença de feridas. A observação da postura, da força e do equilíbrio também são importantes, bem como a palpação dos vários tecidos. Também é recomendável a medição dos membros, avaliando o perímetro e amplitude de movimento, bem como a massa muscular (SILVA, 2008; GOFF, 2016).

A sensibilidade a dor também deve ser avaliada. Entretanto, esta avaliação em animais é algo complexo e subjetivo, uma vez que depende da avaliação do profissional e do proprietário. Existem várias formas de quantificar e qualificar a dor, muitas utilizando observações na mudança comportamental do animal, entretanto, atualmente, são utilizadas escalas unidimensionais como a escala visual analógica (VAS), escala de graduação numérica (NRS), a Escala composta de Glasgow, entre outras (TRANQUILLI et al., 2005; MORAN; HOFMEISTER, 2013). Cada escala tem suas vantagens e desvantagens específicas, mas todas compartilham o ser humano observando o animal e não pelo próprio paciente (MORAN; HOFMEISTER, 2013), assim é recomendável uma associação abrangente, levando em conta a experiência profissional, os dados coletados no exame físico e na anamnese.

Após a avaliação do paciente e ao compreender suas necessidades, poderão ser eleitos os recursos, os equipamentos e as técnicas que isoladas ou combinadas poderão contribuir para a reabilitação física do cão. Os protocolos podem envolver várias modalidades de exercícios e técnicas (DINIZGAMA, 2007).

Uma vez determinado o protocolo, é de extrema importância orientar o proprietário quanto aos cuidados domiciliares e a resposta do cão frente ao tratamento (exemplo, pode ser normal o cão apresentar leve fadiga após a sessão). Também devem ser realizadas reavaliações periódicas do paciente e dos protocolos no decorrer da evolução do tratamento, com o intuito de readequações e correção de respostas indesejáveis (HUMMEL; VICENTE, 2019).

\subsection{Cinesioterapia}

A cinesioterapia é a técnica que realiza os movimentos com o uso de exercícios respeitando uma intensidade, duração e intervalos de tempo (FORMENTON, 2019).

Os objetivos dos exercícios terapêuticos incluem a prevenção das disfunções, a melhora, restauração ou a manutenção da normalidade da força, da mobilidade, da flexibilidade e da coordenação, tanto de forma preventiva como curativa (AMARAL, 2009; FORMENTON, 2019).

Os movimentos utilizados demandam a atividade muscular do paciente ou podem provocar uma resposta muscular aos estímulos resultantes de uma manobra específica. Exercícios proprioceptivos promovem a excitação das terminações nervosas, determinando de maneira automática ou reflexa, o estabelecimento de respostas musculares para que o movimento possa ser aprendido, reabilitado ou reprogramado (AMARAL, 2009).

Os exercícios podem ainda ser classificados em passivo, ativo ou ativo assistido (FORMENTON, 2019). Os exercícios passivos abrangem exercícios conduzidos pelo médico veterinário, sendo utilizado quando o cão tem perda dos movimentos voluntários, tônus e propriocepção, tendo como objetivo a recuperação da deambulação normal. Entre os exercícios passivos se tem o estímulo dos reflexos flexor e extensor, muito utilizado em pacientes com déficit de neurônio motor superior (NMS) (OBLY, 2008).

Os exercícios ativos são os do tipo sustentação assistida, estimulação de propriocepção, esteira, marcha assistida, hidroterapia e outros que ocorrem com o paciente fazendo o movimento sozinho e busca reabilitar cães, que têm o controle dos movimentos presentes, mas se deseja aumentar a massa e força muscular, melhorar a propriocepção, restaurar 
a coordenação motora e controlar a dor (OBLY, 2008; FORMENTON, 2019).

Por último, nos exercícios do tipo ativo assistido, com o animal em estação, ou seja, com os quatro membros apoiados no chão, são realizados movimentos com auxílio do profissional. Várias técnicas podem ser utilizadas com utilização de tipoia ou alças de suspensão apoiadas abaixo do tórax ou em região caudal ao abdômen (MILLIS; LEVINE, 2014), exercícios que estimulam a propriocepção e o equilíbrio, marcha assistida, emprego de esteira ou hidroterapia (ALVES; STURION; GOBETTI, 2019).

As técnicas de alongamentos são mais indicadas para casos de lesão ortopédica, as quais visam aumentar a extensão de músculos, de tendões e de articulações (FORMENTON, 2019).

\subsection{Hidroterapia}

A hidroterapia é um tipo de exercício ativo assistido dentro da água, tem a função de ganho de massa e força muscular, além de mobilizar ativamente as articulações, sem contudo, a aplicação de força direta sobre esses. A água permite a redução da pressão e a carga de peso do animal, ao mesmo tempo em que aumenta o efeito dos esforços por promover resistência ao movimento. Quando utilizado água morna, há estímulo da circulação sanguínea e melhora da drenagem linfática (GORDON-EVANS; KNAP; SCHULZ, 2014; HUMMEL; VICENTE; PESTANA, 2019).

Ao contrário dos exercícios no solo, os aquáticos são executados em baixa velocidade, diminuindo o impacto do movimento (BIASOLI; MACHADO, 2006). A imersão do paciente pode ser total, só o pescoço e a cabeça do cão ficam fora da água e não há apoio dos membros no solo. Já na imersão parcial, o cão apoia os membros no fundo da piscina e se movimenta pela esteira (HUMMEL; VICENTE; PESTANA, 2019).

Pode ser utilizada em diversas patologias, como artroses, patologias da coluna, tratamentos pós-cirúrgicos em ortopedia, displasia coxofemoral, entre outras. Na maior parte dos casos, é utilizada conjuntamente com outras terapias, inclusive, a medicamentosa (MIKAIL, 2009a).

Entretanto, não é indicada para cães com feridas abertas, incontinência urinária, diarreia, problemas cardíacos e respiratórios (MIKAIL, 2009a; ALVES; STURION; GOBETTI, 2019; HUMMEL; VICENTE; PESTANA, 2019). Em animais que sofrem da doença do disco intervertebral (DDIV) cervical deve ser utilizada com cautela, principalmente, em fases mais avançadas do tratamento, sendo contraindicada em casos de dor aguda (MIKAIL, 2009a).

\subsection{Crioterapia}

A crioterapia é baseada no uso de substâncias para retirar o calor corporal sendo indicada em processos dolorosos, edema, perda da função, rubor e hipertermia, porém não é capaz de diminuir o processo inflamatório no local lesionado (LOPES,
2009).

O frio é o agente térmico escolhido para o tratamento da fase aguda de lesão do tecido. Proporciona analgesia e é útil no controle do processo inflamatório pós-cirúrgico, pois atenua os sinais cardinais da inflamação. É um procedimento seguro, entretanto não recomendado em pacientes sensíveis ao frio ou sistemicamente hipotérmicos, imediatamente, após cirurgia (LOPES, 2009; GORDON-EVANS; KNAP; SCHULZ, 2014).

A crioterapia pode ser realizada utilizando bolsas de gelo, diretamente no local afetado, de acordo com a necessidade e protocolo desenvolvido. Existem outras formas de crioterapia, como imersão em banho de gelo, sprays e massagens com gelo em áreas dolorosas ou locais de acupuntura. Deve ter total cuidado para não causar queimaduras, sendo que uma vermelhidão no local de aplicação da técnica é normal. É indicada para a fase aguda de lesão tecidual e após exercícios de reabilitação para diminuir as respostas inflamatórias secundárias (LOPES, 2009).

\subsection{Termoterapia}

A termoterapia apresenta o uso do calor como forma de tratamento no local da lesão. $\mathrm{O}$ calor promove ação vasodilatadora, aumenta a velocidade de condução dos impulsos nervosos, causa relaxamento muscular, aumenta as atividades enzimática e metabólica, bem como a extensibilidade do tecido conectivo, promovendo efeitos sedativos e analgésicos e melhora da extensibilidade de tecido fibroso. Proporciona, ainda, elevação da pressão sanguínea, ventilação pulmonar e pulso GORDON-EVANS; KNAP; SCHULZ, 2014; ALVES; STURION; GOBETTI, 2019).

Este tipo de terapia pode ser aplicada cerca de quatro horas antes da mobilização, estiramento das articulações e dos exercícios terapêuticos, uma vez que aumentam a elasticidade dos tecidos e a mobilidade das articulações (GORDONEVANS; KNAP; SCHULZ, 2014).

Quando necessária a utilização de calor profundo, o tratamento deve ser com o uso de ultrassom terapêutico, no qual as ondas serão absorvidas pelo tecido e a energia mecânica convertida em calor. Esse tratamento é indicado em casos de artrites, bursites, dor, espasmos e afecções musculares e deve ser evitado quando o paciente tem feridas abertas, fases agudas de processo inflamatório, tumores, hematomas e pacientes termossensíveis (STEISS; LEVINE, 2008; ARAÚJO, 2009).

\subsection{Eletroterapia}

A eletroterapia consiste na aplicação de corrente elétrica, de baixa frequência, por meio de eletrodos acoplados na pele do paciente, objetivando a estimulação de nervos periféricos e fibras musculares. É indicada para tratamento de lesões ortopédicas e afecções neurológicas propiciando redução da dor e atrofia muscular. Recomenda-se fazer a tricotomia da região a ser aplicada ou, em caso de impossibilidade, fazer o 
uso abundante do gel. A finalidade do tratamento ditará o tipo do equipamento a usar, sendo Transcutaneal electrical nerve stimulation (TENS), Neuro muscular electrical stimulation (NMES) ou Functional electrical stimulation (FES) (ALVES; STURION; GOBETTI, 2019).

Transcutaneal electrical nerve stimulation - TENS é indicado para o manejo e controle da dor promovendo a analgesia, mediante despolarização de fibras sensitivas. Pode ser utilizado no modo convencional, pulsado ou acupuntura, sendo os dois primeiros mais usados e o terceiro causa mais desconforto ao animal (MIKAIL, 2009b).

Neuro muscular electrical stimulation - NMES é utilizado para tratamento muscular (redução de atrofia), comumente mais utilizado para fortalecer gerando contração tetânica nos músculos por despolarizar o nervo motor (MIKAIL, 2009b). Functional electrical stimulation - FES indicado em casos de paraplegia, paraparesia, atrofia muscular e controle de espasticidade muscular. Nessa funcionalidade realiza a contração de músculos privados do controle nervoso (ALVES; STURION; GOBETTI, 2019).

\subsection{Laser terapêutico (laserterapia)}

Utilizado para promover analgesia e ação antiinflamatória, além de modular diversos processos biológicos, acelerar o tratamento de feridas e articulações e promover a regeneração muscular. O laser acelera a cicatrização por atrair um maior número de fibroblastos, aumentar a produção de colágeno e estímulo da microcirculação (MIKAIL, 2009c; RYOR; MILLIS, 2015).

O laser terapêutico pode ser utilizado de forma isolada ou em combinação com outras técnicas (MIKAIL, 2009c; RYOR; MILLIS, 2015). Pode ser aplicado de forma pontual no local da lesão. Nos casos de pacientes acometidos pela DDIV cervical, o laser é também indicado por promover analgesia com a redução da condução das fibras axonais C (de condução lenta dos impulsos nervosos), responsáveis pela dor crônica persistente (MIKAIL, 2009c).

\subsection{Magnetoterapia}

A magnetoterapia é a aplicação terapêutica de campos magnéticos $(\mathrm{CM})$ através de dois polos produzidos por corrente elétrica, com o objetivo de aumentar o fluxo sanguíneo local, produzindo efeitos anti-inflamatórios (HUMMEL; VICENTE, 2019)).

Podem ser utilizados magnetos estáticos, que geram um $\mathrm{CM}$ contínuo gerando um efeito térmico de relaxamento, ou CM pulsátil (por aparelhos específicos), que gera um menor efeito térmico e mais ação estimulante ou de ativação celular. Estudos sugerem sua utilização na reparação óssea, estimulando o metabolismo do cálcio e, portanto, favorecendo a reparação de fraturas (MIKAIL, 2009d; HUMMEL; VICENTE, 2019).

\subsection{Aparelhos fisioterapêuticos veterinários}

Vê-se, atualmente, modelos cada vez mais aperfeiçoados de cadeiras de rodas para cães, principalmente, com paralisia de membros pélvicos, os aparelhos permitem que os animais se locomovam fazendo apoio com os membros torácicos. Contudo, sua finalidade muitas vezes têm o foco em facilitar a locomoção e não compreende a reabilitação, como consequência pode ser desenvolvida fraqueza e atrofia no membro afetado (DINIZ-GAMA, 2007) se utilizado de forma isolada. Dessa forma, ainda, segundo o autor, novas possibilidades de terapia surgem com os aparelhos fisioterapêuticos veterinários (AFV), que têm a função de promover a reabilitação, principalmente, para casos de tratamento em longo prazo (DINIZ-GAMA, 2007).

O uso de AFV permite o tratamento para a recuperação de movimentos de animais com paresias ou paralisias, minimizar atrofia muscular, diminuir ocorrência de enfermidades respiratórias em função do decúbito prolongado, prevenir sequelas, escaras de decúbito e lesões, além de contribuir assim como as demais modalidades de fisioterapia para maior sobrevida e bem-estar de pacientes com patologias degenerativas. $\mathrm{O}$ aparelho não é recomendado para cães com fraqueza nos membros torácicos (DINIZ-GAMA, 2007).

\section{Conclusão}

A reabilitação física em cães com lesões ortopédicas é um campo fundamental para a promoção do reestabelecimento da função da estrutura lesionada, podendo ser utilizada em período pós-cirúrgico ou aplicada como método conservativo.

Nesse sentido, a fisioterapia se demonstra benéfica no tratamento e prevenção de patologias ortopédicas, neurológicas e também no controle da obesidade canina. Várias técnicas e equipamentos podem ser empregados, sendo o protocolo terapêutico individual, devendo sempre ser elaborado com base nas necessidades e limitações de cada paciente.

Para isso, é imprescindível que o Médico Veterinário Fisiatra tenha pleno conhecimento das técnicas de reabilitação.

\section{Referências}

ANDRADES, A.O. et al. Modalidades fisioterapêuticas na reabilitação de cães com doença do disco intervertebral toracolombar submetidos à cirurgia descompressiva: 30 casos (2008-2016). Arq. Bras. Med. Vet. Zootec., v. 70, n. 4, p. 10891098, 2018.

ALVES, M.V.L.D.; STURION, M.A.T.; GOBETTI, S.T.C. Aspectos gerais da fisioterapia e reabilitação na medicina veterinária. Ciênc. Vet. UniFil, v.1, n.3, p.69-78, 2019.

AMARAL, A.B. Cinesioterapia. In: MIKAIL, S.; PEDRO, C. R. Fisioterapia veterinária. Barueri: Manole, 2009. p.49-62.

ANDRADES, A.O. et al. Modalidades fisioterapêuticas na reabilitação de cães com doença do disco intervertebral toracolombar submetidos à cirurgia descompressiva: 30 casos (2008-2016). Arq. Bras. Med. Vet. Zootec., v.70, n.4, p.10891098, 2018.

ARAUJO, M.A. Termoterapia. In: MIKAIL, S.; PEDRO, C. 
Fisioterapia veterinária. Barueri: Manole, 2009. p.76-88.

BIASOLI, M. C.; MACHADO, C. M. C. Hidroterapia: aplicabilidades clínicas. Rev. Bras. Med. v.63, n.5, p.225-237, 2006.

CAMPOS, M. G. et al. A fisioterapia no tratamento das sequelas de cinomose. Ciênc. Animal, v.30, n.1, p.154-161, 2020.

DINIZ-GAMA, E.J. Perfil clínico dos animais e funcionalidade do uso do aparelho de fisioterapia veterinária (modelos vetcar) na reabilitação de cães e gatos acometidos por dificuldades de locomoção. 2007. 85 f. Dissertação (mestrado) - Universidade Estadual Paulista, Faculdade de Medicina Veterinária e Zootecnia, 2007.

EUGÊNIO, F.R. Semiologia do Sistema Locomotor em Cse, ou util. In.: FEITOSA, F.L.F. Semiologia veterinária: a arte do diagnóstico. São Paulo: Roca, 2014. p.1047-1091.

FORMENTON, M.R. Cinesioterapia. In: HUMMEL, J.; VICENTE, G. Tratado de fisioterapia e fisiatria de pequenos animais. São Paulo: Payá, 2019. p.38-53.

GOFF, L. Physiotherapy assessment for animals. In.: McGOWAN, C.M.; GOFF, L Animal physiotherapy: m. assessment, treatment and rehabilitation of animals. Oxfordo: Blackwell, 2007. p.171211.

GORDON-EVANS, W.; KNAP, K.; SCHULZ, K. S. Fundamentos da reabilitação física. In: FOSSUM, T.W. Cirurgia de pequenos animais. Rio de Janeiro: Elsevier, 2014. p.114-130.

HUMMEL, J.; VICENTE, G; PESTANA, N.S. Tratado de fisioterapia e fisiatria de pequenos animais. São Paulo: Payá, 2019.

LOPES, A. D. Crioterapia. In: MIKAIL, S.; PEDRO, C. R. Fisioterapia veterinária. Barueri: Manole, 2009. p.66-70.

MARCONDES, N. Semiologia do sistema nervoso de pequenos animais. In: FEITOSA, F.L.F. Semiologia veterinária: a arte do diagnóstico. São Paulo: Roca, 2014. p.812- 895.

MIKAIL, S.; PEDRO, C. R. Fisioterapia veterinária. Barueri:
Manole, 2009b.

MIKAIL, S. Hidroterapia. In.: MIKAIL, S.; PEDRO, C.R. Fisioterapia veterinária. Barueri: Manole, 2009a. p.71-75.

MIKAIL, S. Eletroterapia. In: MIKAIL, S.; PEDRO, C. (Ed.). Fisioterapia veterinária. Barueri: Manole, 2009b, p.103-109.

MIKAIL, S. Laser Terapêutico. In: MIKAIL, S.; PEDRO, C. (Ed.). Fisioterapia veterinária. Barueri: Manole, 2009c. p.89-97.

MIKAIL, S. Magnetoterapia. In: MIKAIL, S.; PEDRO, C. (Ed.). Fisioterapia veterinária. Barueri: Manole, 2009d. p.98-101.

MORAN, C.E.; HOFMEISTER, E.H. Prevalence of pain in a university veterinary intensive care unit. J. Vet. Emerg. Critical Care, v.23, n.1, p.29-36, 2013.

MUCHA, M.; BOCKSTAHLER, B. Physiotherapy as a possibility to increase quality of life in old dogs. 6th VEPRA - 1ST ECVSMR CONFERENCE 2019, Ghent, 19th - 21st September. Small Animal Program. Belgica. p.51 - 52.

OBLY, N. Reabilitação neurológica. In: TAYLOR, R. et al. (Ed.). Reabilitação e fisioterapia na prática de pequenos animais. São Paulo: Roca, 2008. p.157-180.

PRYOR, B.; MILLIS, D.L. Therapeutic laser in veterinary medicine. Vet Clin North Am Small Anim Pract., v. 45, n. 1, p. 45-56, 2015.

SIMS, C.; WALDRON, R.; MARCELLIN-LITTLE, D.J. Rehabilitation and physical therapy for the neurologic veterinary patient. Vet Clin North Am Small Anim Pract., v.45, n.1, p.123$143,2015$.

STEISS, J.E.; LEVINE, D. Modalidades de agentes físicos. In: TAYLOR, R. et al. (Ed.). Reabilitação e fisioterapia na prática de pequenos animais: ROCA, 2008. p.75-94.

TRANQUILLI, W.J.; GRIMM, K.A.; LAMONT, L.A. Terminologia, fisiologia, reconhecimento e estratégias clínicas para tratamento da dor. In: Tratamento da dor para o clínico de pequenos animais. São Paulo: Roca, 2005. p. 1-12. 